\title{
Trends in Resistance to Extended-Spectrum Cephalosporins and Carbapenems among Escherichia coli and Klebsiella spp. Isolates in a District in Western India during 2004-2014
}

\author{
Ingvild Odsbu ${ }^{1, *,+}+{ }^{(1)}$, Smita Khedkar ${ }^{2,+}{ }^{,}$Frida Lind ${ }^{1}$, Uday Khedkar ${ }^{2}$, Sandeep S. Nerkar ${ }^{1,3}$, \\ Nicola Orsini ${ }^{1}$, Ashok J. Tamhankar 1,3 (i) and Cecilia Stålsby Lundborg 1 (i) \\ 1 Department of Public Health Sciences, Karolinska Institutet, 17177 Stockholm, Sweden; \\ adirf81@hotmail.com (F.L.); san.ner1978@gmail.com (S.S.N.); nicola.orsini@ki.se (N.O.); \\ ejetee@gmail.com (A.J.T.); cecilia.stalsby.lundborg@ki.se (C.S.L.) \\ 2 Bac-Test Laboratory, College Road, Nashik 422005, Maharashtra, India; mail@bactestlab.com (S.K.); \\ udayk_nsk@sancharnet.in (U.K.) \\ 3 Indian Initiative for Management of Antibiotic Resistance, Department of Environmental Medicine, \\ R.D. Gardi Medical College, Ujjain 456006, India \\ * Correspondence: ingvild.odsbu@ki.se; Tel.: +46-(0)-8-524-833-81 \\ + These authors contributed equally to this work.
}

Received: 9 December 2017; Accepted: 16 January 2018; Published: 19 January 2018

\begin{abstract}
Surveillance data on the level of resistant bacteria is needed to inform strategies to reduce the development and spread of antibiotic resistance. The aim of this study was to determine the non-susceptibility trends to extended-spectrum cephalosporins and carbapenems among Escherichia coli and Klebsiella spp. isolates from the district of Nashik in Western India during the period 2004-2014. Antibacterial susceptibility testing of clinical isolates was performed using Kirby-Bauer disc diffusion method to determine inhibitory zone diameters. The change in proportions of non-susceptible bacteria over calendar time was investigated with spline transformations in a logistic regression model. For the extended-spectrum cephalosporins, the proportions of non-susceptible E. coli and Klebsiella spp. isolates were above $78.4 \%$ and $84.9 \%$ throughout the study period, respectively. E. coli and Klebsiella spp. isolates exhibited carbapenem non-susceptibility levels as high as $76.9 \%$ and $84.1 \%$ respectively. The proportions of extended-spectrum betalactamase (ESBL)-producing isolates ranged from 38.3-85.9\% in E. coli and from 45.1-93.1\% in Klebsiella spp. Significantly higher proportions of non-susceptible and ESBL-producing isolates were found among isolates from inpatients compared to isolates from outpatients for both E. coli and Klebsiella spp. $(p<0.050)$. The high proportions of non-susceptible isolates observed show that there is great need to focus on optimal use of antibiotics to reduce the development of antibiotic resistance.
\end{abstract}

Keywords: Escherichia coli; Klebsiella; India; ESBL; cephalosporins; carbapenems; antibiotic resistance; non-susceptible; antimicrobial susceptibility testing

\section{Introduction}

The increase in antibiotic resistance has been acknowledged as one of the top three greatest threats to global health in our time [1]. In 2014, the World Health Organization (WHO) published a report on the global surveillance of antimicrobial resistance [2]. Mapping of the availability of national surveillance data on antimicrobial resistance worldwide showed large gaps, particularly in Africa and South and South-East Asia, which is of great concern. Without such data, we will not know the scope of the problem, we will not know where to put our efforts and we will not be able to detect new trends 
and threats. In the Global Action Plan on Antimicrobial Resistance (GAP-AMR, with special emphasis on antibiotic resistance) adopted by the World Health Assembly in May 2015, one of the objectives is to strengthen the knowledge and evidence base through surveillance and research [3]. As a response to the GAP-AMR, the WHO has launched an initiative called the Global Antimicrobial Resistance Surveillance System (GLASS) that aims to establish a global standardized approach to the collection of, analysis and sharing of data [4]. Setting up a national surveillance system can be a challenge to many countries, particularly in countries where health systems are weak. In addition to the costs of running a laboratory of high standards, there is a need for skilled personnel and standardized methods that allow for comparisons of resistance proportions between countries.

Since a national surveillance system is lacking in India, there is a great need for long-term studies on the proportions and trends in antibiotic resistance. Several long-term studies from India have found high antibiotic resistance proportions, but most of these studies are single-site studies or cover shorter periods of time [5-11]. Recently, a retrospective study over seven years from a private laboratory network in India concluded that there are high proportions of resistant bacterial strains among blood culture isolates from patients across India [12].

High proportions of resistant Gram-negative bacterial strains are of great concern since there are few treatment options left, especially for the extended-spectrum cephalosporin and carbapenem-resistant E. coli and Klebsiella spp. strains. In this study, we have examined the trends in resistance to extended-spectrum cephalosporins and carbapenems among clinical E. coli and Klebsiella spp. isolates in the district of Nashik in Western India over an 11-year period (2004-2014).

\section{Materials and Methods}

\subsection{Study Setting}

The data used in this study were primarily collected for antibacterial susceptibility testing of clinical bacterial isolates and secondarily for surveillance and research purposes. The antibacterial susceptibility testing was performed at the Bac-Test Laboratory, an International Organization for Standardization (ISO) certified laboratory that serves various hospitals, clinics and diagnostic laboratories in the district of Nashik [13], which belongs to the state of Maharashtra in the western part of India and has a population of more than 6.1 million and covers an area of 15,582 sq km [14].

\subsection{Study Material}

Information about the patient (gender), type of patient (in- or outpatient), type of specimen (urine, blood etc.), date of specimen collection, type of bacterial species and the results of the antibacterial susceptibility testing for every isolate were recorded in the WHONET software [15]. The WHONET is a free of charge software program developed by the WHO to enable collection of susceptibility data in resource-poor settings $[15,16]$. Information about several isolates (e.g., different year, different specimen types, and/or different species) may have been obtained from the same patient. The data were collected in the period from 1 January 2004 to 31 December 2014. All years contained data for every month of the year, except for 2010 which contained data for January only (hence the sample size is small $(n<30)$ ). The data file for the rest of 2010 was corrupted at the laboratory and could not be revived. Type of patient (in- or outpatient) was only available for the period 2011-2014.

The data material used in this study was retrospective data from routine analysis of antibacterial susceptibilities. The data were anonymous since no patient identifiers were available, and it would therefore not be possible to obtain informed consent from the patients.

\subsection{Laboratory Methods}

Antibiotic susceptibility testing was performed using the Kirby-Bauer disk diffusion method (with disks from HiMedia Laboratories, Mumbai, India and Becton, Dickinson, Franklin Lakes, NJ, USA) according to the most updated Clinical and Laboratory Standards Institute (CLSI) guideline at 
the time of analysis $[17,18]$. The double disk synergy test was used to screen for extended-spectrum betalactamase (ESBL)-producing bacterial strains [19]. Some of the ESBL positive isolates may not possess antibacterial resistance genes, hence, the term "ESBL-positive" used here reflects an ESBL phenotype-positive isolate. The decision on which panel of antibiotics to test for a particular isolate was based on type of bacterial strain, type of specimen (different infections require different panels of antibiotics to be tested), and sometimes specific requirements of the clinicians.

\subsection{Statistical Analyses}

For analyses purposes we interpreted the recorded inhibitory zone diameters according to the clinical zone diameter breakpoints provided by the CLSI guideline used at the time of the antibacterial susceptibility testing [19]. Susceptible bacterial isolates were classified as "susceptible", whereas intermediate susceptible and resistant bacterial isolates were classified as "non-susceptible". The antibacterial categories included in the study were extended-spectrum cephalosporins (cefepime, cefixime, cefotaxime, cefpodoxime, and ceftazidime) and carbapenems (meropenem and imipenem). Non-susceptibility to an antibacterial category was defined as being non-susceptible to at least one antibacterial agent in an antibacterial category [20]. Changes in the CLSI guidelines for clinical zone diameter breakpoints for cefotaxime and ceftazidime in 2010, meropenem and imipenem in 2011, and cefepime in 2014 were accounted for when classifying bacterial isolates as "susceptible" or "non-susceptible".

Proportions of non-susceptible bacterial isolates (number of non-susceptible isolates as a proportion of total isolates tested) were calculated from the antibacterial susceptibility data. Calendar time (year) was flexible modelled as a continuous predictor using regression splines in two ways. First, a principled approach known as multivariable regression spline approach (MVRS) [21], was used to flexibly smooth the trajectory over time without imposing any constraints on the functional relation. The MVRS selected the model that had the best fit of the data using the smallest number of parameters. Second, a visual inspection of the calculated proportions of non-susceptible isolates over time was done by the authors and along with the predicted smooth trends these two approaches provided the basis to identify the most likely point in time where the outcome may have changed (knot). For its simplicity, we approximated the trajectories of proportions of non-susceptible bacterial isolates over calendar time with two linear trends, one before the selected knot and one after the selected knot. Odds ratios (ORs) of non-susceptible bacterial isolates associated with 1-year increment before and after the knot were estimated along with 95\% confidence intervals using piece-wise linear splines. Calculated proportions of non-susceptible isolates were compared between inpatients and outpatients using Chi square test. A two-sided $p$-value $<0.05$ was considered statistically significant. Statistical analyses were performed using Stata version 14 (Stata Corp, TX, USA).

\section{Results}

\subsection{Bacterial Isolates}

The data set contained information about 9813 clinical isolates collected during the period 2004-2014 out of which 5863 isolates were identified as E. coli, and 3950 isolates were identified as Klebsiella spp. The most common specimen types for E. coli isolates were urine (56.6\%), stool (18.5\%) and pus (12\%). For Klebsiella spp. isolates the most common specimen types were urine $(31.1 \%)$, from the respiratory tract $(21.0 \%)$ and pus $(20.6 \%)$. The proportions of isolates from blood were $2.3 \%$ and $10.8 \%$ for E. coli and Klebsiella spp., respectively. The isolates originated to a slightly higher extent from males $(58.2 \%)$ compared to females $(41.8 \%)$ for Klebsiella spp., while no sex differences were observed for E. coli isolates. About $60 \%$ of the E. coli isolates and $70 \%$ of the Klebsiella spp. isolates came from inpatients, and these proportions were consistent for every year in the period during which this information was available (2011-2014). 


\subsection{Trends in Non-Susceptible E. coli and Klebsiella spp. Isolates during the Period 2004-2014}

For the E. coli isolates, $98.3 \%(n=5764)$ were tested for susceptibility to extended-spectrum cephalosporins and $77.8 \%(n=4564)$ were tested for susceptibility to carbapenems. For the Klebsiella spp. isolates, $98.1 \%(n=3873)$ were tested for susceptibility to extended-spectrum cephalosporins and $87.7 \%(n=3463)$ were tested for susceptibility to carbapenems. High proportions of non-susceptible E. coli and Klebsiella spp. isolates were found for the extended-spectrum cephalosporins throughout the period, ranging from 78.4 to $100 \%$ and from 84.9 to $100 \%$, respectively (Figure 1 and Table A1).

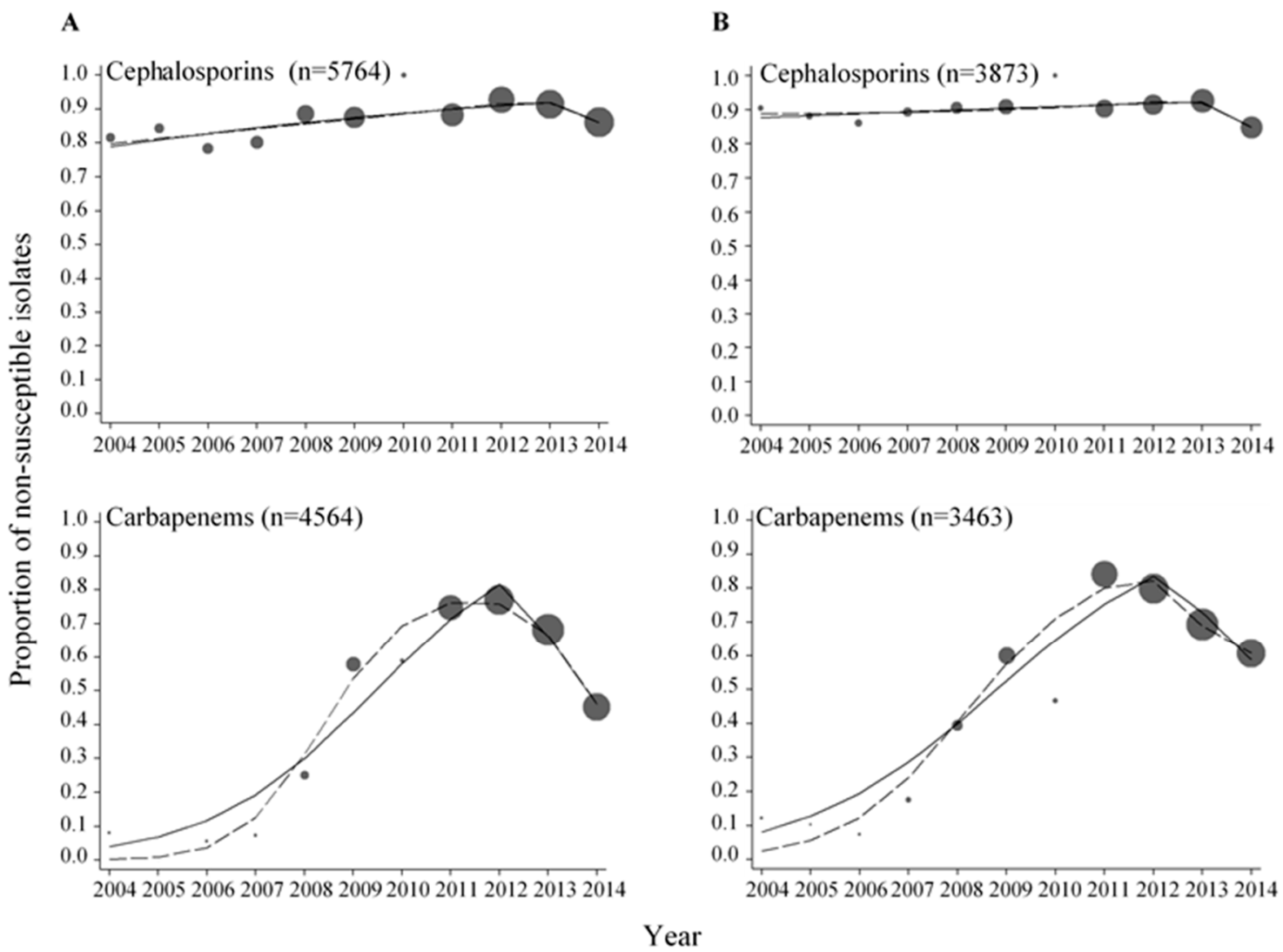

Figure 1. Proportions of E. coli (A) and Klebsiella spp. (B) isolates non-susceptible to cephalosporins and carbapenems. The data were fitted with a piece-wise linear logistic regression model (solid line) and multivariable regression spline procedure (MVRS) (dashed line). Each bubble shows the proportion of non-susceptible isolates to the specific antibacterial category for the years 2004-2014. The size of the bubbles represents the number of isolates tested per year relative to the total number of isolates tested ( $n$ ) (see Table A1). $n=$ total number of isolates tested for the specific antibacterial category in the whole period.

We next analyzed trends in proportions of non-susceptible isolates using splines of calendar time. The year 2013 was identified as the year where a change in the proportions of extended-spectrum cephalosporin non-susceptible E. coli and Klebsiella spp. isolates most likely occurred (knot year) (Table 1). Per every year increment in the period 2004-2013 there was a 13\% increase in the odds of an E. coli isolate being non-susceptible to extended-spectrum cephalosporins, whereas in the period 2013-2014 there was a 52\% decrease in the odds of an E. coli isolate being non-susceptible. For Klebsiella spp., there was a $6 \%$ increase in the odds of an isolate being non-susceptible to extended-spectrum cephalosporins per every year increment in the period 2004-2013, whereas in the period 2013-2014 there was a 56\% decrease in the odds of an isolate being non-susceptible. Hence, for both E. coli and Klebsiella spp. there was an increasing trend in the proportions of non-susceptible 
isolates before 2013 and a decreasing trend in the proportions of non-susceptible isolates after 2013. For the carbapenems, the proportions of non-susceptible isolates ranged from 0 to $76.9 \%$ in E. coli and from 7.4 to $84.1 \%$ in Klebsiella spp., respectively (Figure 1 and Table A1). Increasing trends in the proportions of carbapenem non-susceptible E. coli and Klebsiella spp. isolates were found before 2012 (knot year) and decreasing trends were found after 2012 (Table 1).

Table 1. Odds ratios (OR) with 95\% confidence intervals (CI) of non-susceptible isolates for every year increase in the period 2004-2014 (2006-2014 for ESBL-producing isolates) from a piece-wise logistic regression model.

\begin{tabular}{|c|c|c|c|c|c|c|}
\hline \multirow{3}{*}{ Organism } & \multirow{3}{*}{$\begin{array}{l}\text { Antibacterial } \\
\text { Category/ESBL }\end{array}$} & \multicolumn{5}{|c|}{ Linear Trend } \\
\hline & & \multicolumn{2}{|c|}{ Before the Knot } & \multirow{2}{*}{$\begin{array}{l}\text { Knot } \\
\text { Year }\end{array}$} & \multicolumn{2}{|c|}{ After the Knot } \\
\hline & & OR & $95 \% \mathrm{CI}$ & & OR & $95 \% \mathrm{CI}$ \\
\hline \multirow{3}{*}{ E. coli } & Cephalosporins & 1.13 & $1.09-1.17$ & 2013 & 0.48 & $0.38-0.61$ \\
\hline & Carbapenems & 1.80 & $1.71-1.89$ & 2012 & 0.24 & $0.21-0.27$ \\
\hline & ESBL-positive & 1.23 & $1.11-1.37$ & 2009 & 0.66 & $0.58-0.76$ \\
\hline \multirow{3}{*}{ Klebsiella spp. } & Cephalosporins & 1.06 & $1.01-1.11$ & 2013 & 0.44 & $0.33-0.59$ \\
\hline & Carbapenems & 1.66 & $1.57-1.75$ & 2012 & 0.32 & $0.28-0.37$ \\
\hline & ESBL-positive & 1.48 & $1.29-1.71$ & 2009 & 0.55 & $0.46-0.66$ \\
\hline
\end{tabular}

ESBL: extended-spectrum betalactamase.

Significantly higher proportions of extended-spectrum cephalosporin and carbapenem non-susceptible isolates were found in inpatients when comparing non-susceptibility rates among isolates from inpatients and outpatients for both E. coli and Klebsiella spp. $(p<0.050$, Table 2). When comparing the trends in proportions of non-susceptible E. coli and Klebsiella spp. isolates during the period 2011-2014, there were no major differences observed among isolates from inpatients compared to isolates from outpatients (Figures A1 and A2, Tables A2 and A3). Although some statistically significant changes were observed (Table A3), particularly among isolates from outpatients, a longer time period than four years would be required to allow for more robust analyses of the trends.

Table 2. Proportions (\%) of non-susceptible or ESBL-producing isolates from inpatients and outpatients according to antibacterial category and ESBL production in the period 2011-2014.

\begin{tabular}{ccccccc}
\hline Antibacterial & \multicolumn{3}{c}{ E. coli } & \multicolumn{3}{c}{ Klebsiella spp. } \\
\cline { 2 - 7 } Category/ESBL & Inpatients & Outpatients & $\boldsymbol{p}^{\mathbf{1}}$ & Inpatients & Outpatients & $\boldsymbol{p}^{\mathbf{1}}$ \\
\hline Cephalosporins & 91.6 & 87.5 & $<0.001$ & 93.4 & 85.8 & $<0.001$ \\
Carbapenems & 67.9 & 58.4 & $<0.001$ & 78.8 & 62.0 & $<0.001$ \\
ESBL-positive & 46.7 & 36.9 & $<0.001$ & 72.5 & 51.2 & $<0.001$ \\
\hline
\end{tabular}

${ }^{1} p<0.050$ (Chi square test, 2-tailed) indicates statistical significance of the differences in non-susceptibility rates. ESBL: extended-spectrum betalactamase.

When comparing non-susceptibility rates among urinary samples (the most common specimen type) and all other specimen types, significantly lower proportions of extended-spectrum cephalosporin and carbapenem non-susceptible isolates were found among urinary samples for both E. coli and Klebsiella spp. $(p<0.050$, Table A4).

Significantly higher proportions of extended-spectrum cephalosporin and carbapenem non-susceptible isolates were found among males compared to females $(p<0.050$, Table A5).

\subsection{Trends in ESBL-Producing E. coli and Klebsiella spp. Isolates during the Period 2006-2014}

Of the 5589 E. coli isolates analysed during the period 2006-2014, 4915 isolates (87.9\%) were tested for ESBL. The proportions of ESBL-producing E. coli ranged from 38.3 to 85.9\% (Figure 2A and 
Table A1) during these years. Per every year increment in the period 2006-2009, there was a $23 \%$ increase in the odds of E. coli isolates being ESBL-positive, whereas in the period 2009-2014 there was a $34 \%$ decrease in the odds of E. coli isolates being ESBL-positive (Table 1). For Klebsiella spp., 3422 out of 3810 isolates (89.8\%) were tested for ESBL in the period 2006-2014. The proportions of ESBL-producing Klebsiella spp. ranged from 45.1 to $93.1 \%$ (Figure 2B and Table A1). As for ESBL-producing E. coli isolates, an increasing trend in the proportions of ESBL-producing Klebsiella spp. was observed before 2009 and a decreasing trend was observed after 2009 (Table 1).

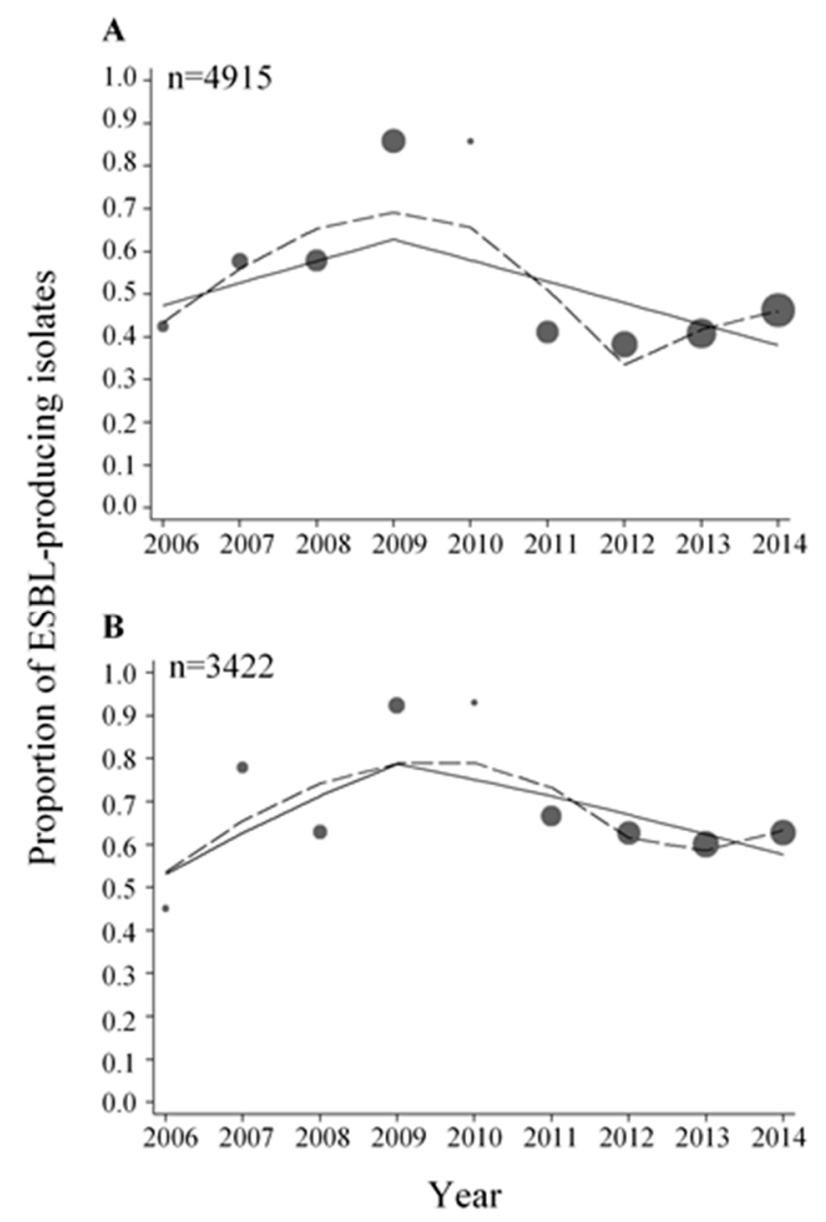

Figure 2. Proportions of ESBL-producing E. coli (A) and Klebsiella spp. (B) isolates. The data were fitted with a piece-wise linear logistic regression model (solid line) and multivariable regression spline procedure (MVRS) (dashed line). Each bubble shows the proportion of ESBL-producing E. coli and Klebsiella spp. isolates for the years 2006-2014. The size of the bubbles represents the number of isolates tested per year relative to the total number of isolates tested ( $n$ ) (see Table A1). $n=$ total number of isolates tested for ESBL in the whole period.

Significantly higher proportions of ESBL-producing isolates were found in inpatients when comparing proportions of ESBL-positive isolates from inpatients and outpatients for both E. coli and Klebsiella spp. $(p<0.050$, Table 2). No major differences in trends were observed among isolates from inpatients compared to isolates from outpatients in the period 2011-2014 (Figures A1 and A2, Tables A2 and A3).

When comparing the proportions of ESBL-producing isolates from urinary samples and all other specimen types, significantly lower proportions of ESBL-producing isolates were found among urinary samples for both E. coli and Klebsiella spp. ( $p<0.050$, Table A4).

Significantly higher proportions of ESBL-producing isolates were found among males compared to females $(p<0.050$, Table A5). 


\section{Discussion}

We have examined data on antibacterial susceptibility over an 11-year period among E. coli and Klebsiella spp. isolates from community- and hospital-acquired infections in the district of Nashik in Western India. High proportions of extended-spectrum cephalosporin and carbapenem non-susceptible isolates, as well as high proportions of ESBL-producing isolates were found for both E. coli and Klebsiella spp. In our data, we observed that the proportions of non-susceptible bacterial isolates did not show a steady increase or decrease over time. Rather, the proportions fluctuated over time. This has also been observed in other long-term studies of trends in antibiotic resistance [5,22-24] and might reflect differences in the annual burden of infections, changes over time in the choice of empiric drugs for treatment of infections and changes in the study population.

We found that the proportions of non-susceptible isolates were higher in inpatients compared to outpatients. Higher prevalence of resistance among bacterial isolates from inpatients compared to outpatients has also been found in other studies [25,26]. Higher prevalence of resistance was also found among bacterial isolates from males compared to females. The differences observed between males and females could be due to differences in exposure to bacterial pathogens and/or antibiotics. Differences in the proportions of non-susceptible isolates were also found when comparing urinary samples and all other specimen types for both E. coli and Klebsiella spp. Lower proportions of non-susceptible isolates from urinary samples might reflect that cephalosporins and carbapenems are not recommended as first line treatment of urinary tract infections. Despite lower proportions of non-susceptible isolates among urinary samples compared to other specimen types, the proportions are alarmingly high. Analysis of nitrofurantoin non-susceptible E. coli isolates during the period 2004-2014 revealed that the non-susceptibility proportions ranged from $4.9-39.8 \%$ (data not shown). The lower prevalence of resistant $E$. coli isolates observed for nitrofurantoin, one of the drugs recommended as first line treatment of urinary tract infections [27], compared to cephalosporins and carbapenems might indicate irrational use of antibiotics to treat urinary tract infections.

To analyse the trends in proportions of non-susceptible isolates, we modelled calendar time using two different approaches, namely a multivariable regression spline model to flexibly model time without constraints and a piece-wise linear spline to summarize the overall change of non-susceptibility over time. Using these approaches, we were able to detect a point in time where a change in the proportions of non-susceptible isolates most probably occurred. In some cases, the MVRS approach indicated complicated trends with probably more than a single point of change in slope. For example, for ESBL-producing E. coli and Klebsiella spp. (Figure 2), the year 2009 was chosen as the knot year (Table 1) resulting in an increasing trend in ESBL-producing isolates before 2009 and a decreasing trend after 2009. From the graph showing the predicted proportions from the MVRS approach, an increasing trend was predicted up to the year 2009, followed by a decreasing trend to the year 2012 in E. coli and 2012-2013 in Klebsiella spp., and then an increasing trend from 2012 and 2013, respectively. It would be possible to choose more than one knot to detect more than one change in the trend, but we wanted to grasp the major changes and therefore chose to use one knot in our analyses.

The years 2009, 2012, and 2013 were identified as the years where a change in the odds of bacterial isolates being non-susceptible or ESBL-producing most likely occurred (Table 1). In the period following these years, decreasing trends in non-susceptible and ESBL-producing isolates were detected for both E. coli and Klebsiella spp. Since we did not have data on antibiotic consumption, neither from the individual patient nor at the aggregated level from the district of Nashik, we can only speculate about the reason for this observation. The WHO selected antimicrobial resistance as theme for World Health Day 2011 [28], and in accordance to this activity directed towards physicians were conducted in the district of Nashik in order to promote rational use of antibiotics. This was done through the Indian Initiative of Management of Antibiotic Resistance (IIMAR) [29]. IIMAR activities were continued further in the program of the Antibiotic Stewardship Network in India [30] in 2013-2014 in the Nashik district. These activities were focusing on proper use of antibiotics, particularly prescription of last resort antibiotics, like carbapenems, to treat infections caused by Gram-negative bacteria (unpublished 
information). Many factors influence prescribing decisions, and raising awareness and knowledge among physicians have been shown to be important to improve the prescribing of antibiotics [31,32]. The decreasing trends in non-susceptible and ESBL-producing isolates observed in the last part of the study period might be attributed to these activities, and also to the increased focus on antibiotic resistance in general, both in India and the rest of the world [2,27,33].

High proportions of extended-spectrum cephalosporin and carbapenem non-susceptible isolates were observed in this study. For the extended-spectrum cephalosporins, the proportions of non-susceptible E. coli and Klebsiella spp. isolates were above $78.4 \%$ and $84.9 \%$, respectively, throughout the period 2004-2014. For the carbapenems, there were much more variations in the proportions of non-susceptible E. coli and Klebsiella spp. isolates. Most samples were collected from 2008 and onwards which reflects the increasing use of carbapenems in the recent years due to increased availability and reduced cost of these drugs (unpublished information). Similar proportions of extended-spectrum cephalosporin and carbapenem non-susceptible isolates have been reported in other studies from India $[10,12,34]$. The high proportions of inpatients (or only inpatients) in the study populations can explain the high proportions of resistance observed in these studies. A study on the proportions of resistance in bacteria isolated from urine in individuals in the community in Northern India revealed low proportions for extended-spectrum cephalosporins, and no resistance towards carbapenems [35].

The proportions of extended-spectrum cephalosporin and carbapenem non-susceptible isolates found in this study are alarmingly high. In Europe, where most countries have national surveillance systems, the population-weighted mean resistance percentages in 2014 were $12 \%$ for extended-spectrum cephalosporins and $<0.1 \%$ for carbapenems among invasive $E$. coli isolates. Among invasive Klebsiella spp. isolates the percentages were $28 \%$ and $7.3 \%$, respectively [36]. Like in India there are variations in the proportions of resistance reported by various regions of Europe, with highest resistance proportions reported from southern and south-eastern Europe.

Of particular concern are the high proportions of ESBL-producing isolates found both in E. coli and Klebsiella spp. High proportions of ESBL-producing bacteria and a rapid increase in recent years have also been observed in other studies from India $[5-7,37,38]$. The high proportions found are worrisome since there are few treatment options left to treat infections caused by these bacteria, particularly if there is also resistance to carbapenems.

\section{Strengths and Limitations}

The data analysed in this study are unique, since longitudinal surveillance data from India are scarce. The data were collected over an 11-year period and analysed at the same laboratory. Although there could be changes in staff and laboratory practices over time, the fact that the same laboratory performed all antibacterial susceptibility testing minimizes the risk for bias due to methodological issues. The availability of data on type of patient made it possible to distinguish between inpatients and outpatients in the period 2011-2014. The use of regression splines instead of using the overall linear trend allowed for more robust analysis of the trends in non-susceptibility over time. A limitation to our study is that a large proportion of the data was collected during the second half of the study period, making the estimates for the first half of the period less robust compared to estimates from the second half of the study period. No patient identifiers were available in the data set meaning that the number of patients included in the study was not known.

\section{Conclusions}

This study highlights the high proportion of antibiotic resistance in India and the need for a national surveillance system. In addition, there is great need to focus on optimal use of antibiotics as well as infection prevention and control measures to combat the emerging threat of antibiotic resistance. Although decreasing trends in antibiotic resistance among E. coli and Klebsiella spp. isolates were observed in recent years, it cannot be ruled out that the trends might increase again which shows the importance of longitudinal surveillance data to detect changes in antibiotic resistance trends. 
Author Contributions: Ingvild Odsbu, Smita Khedkar, Frida Lind, Uday Khedkar, Sandeep S. Nerkar, Ashok J. Tamhankar, Nicola Orsini and Cecilia Stålsby Lundborg conceived and designed the study; Smita Khedkar and Uday Khedkar performed the experiments; Ingvild Odsbu, Smita Khedkar, Frida Lind, Uday Khedkar, Sandeep S. Nerkar, Ashok J. Tamhankar, Nicola Orsini and Cecilia Stålsby Lundborg analyzed the data; Smita Khedkar, Uday Khedkar and Cecilia Stålsby Lundborg contributed reagents/materials/analysis tools; Ingvild Odsbu, Smita Khedkar, Frida Lind, Uday Khedkar, Sandeep S. Nerkar, Ashok J. Tamhankar, Nicola Orsini and Cecilia Stålsby Lundborg wrote the paper.

Conflicts of Interest: The authors declare no conflict of interest.

\section{Appendix}

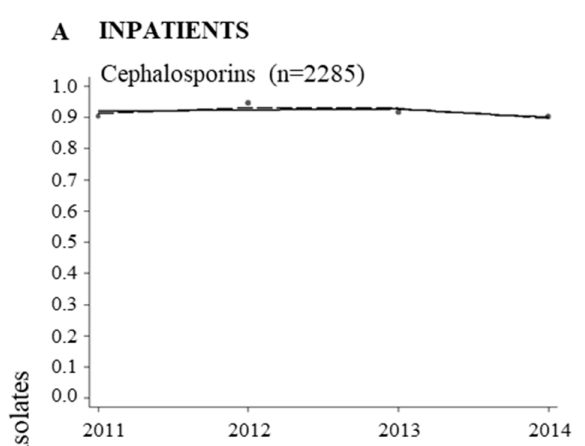

\section{B OUTPATIENTS}
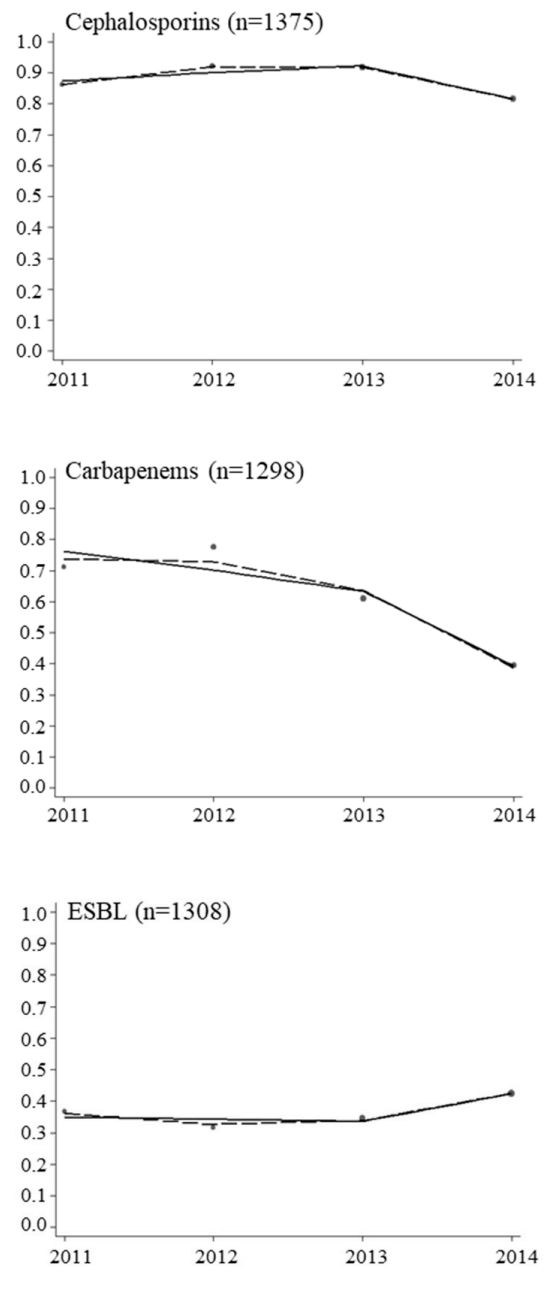

Figure A1. Proportions of E. coli isolates non-susceptible to cephalosporins and carbapenems, and extended-spectrum betalactamase (ESBL)-producing isolates from inpatients (A) and outpatients (B). The data were fitted with a piece-wise linear logistic regression model (solid line) and multivariable regression spline procedure (MVRS) (dashed line). Each bubble shows the proportion of isolates non-susceptible to the specific antibacterial category or the proportion of ESBL-producing isolates for the years 2011-2014. The size of the bubbles represents the number of isolates tested per year relative to the total number of isolates tested ( $n$ ) (see Table A2). $n=$ total number of isolates tested for the specific antibacterial category or ESBL-production in the whole period. 


\section{A INPATIENTS}

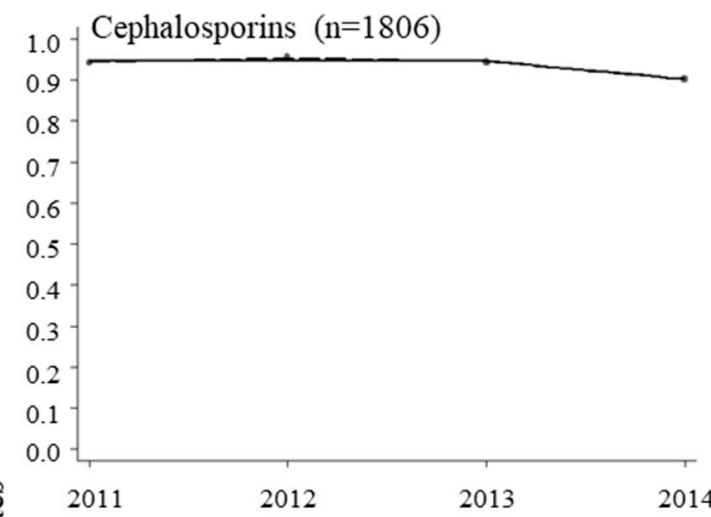

\section{B OUTPATIENTS}

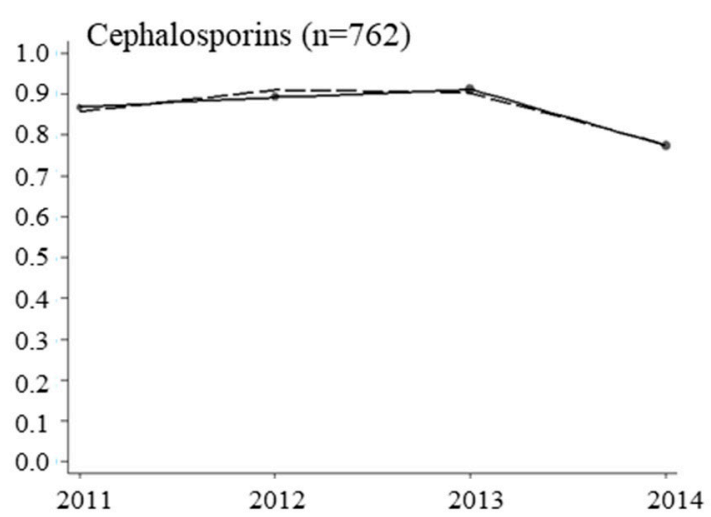

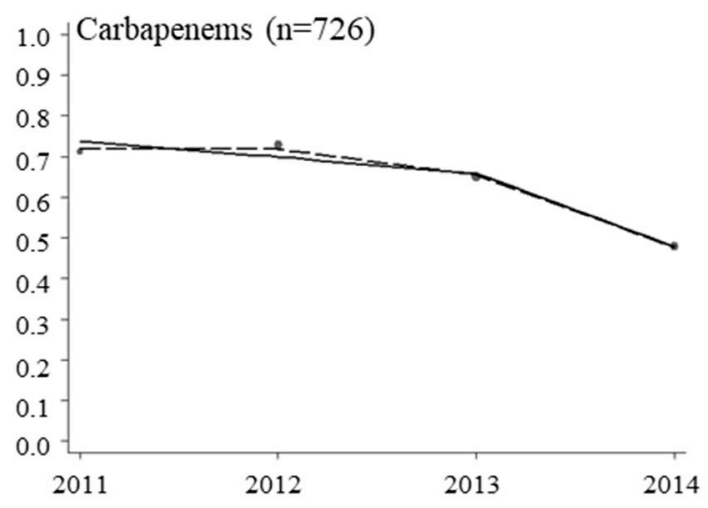

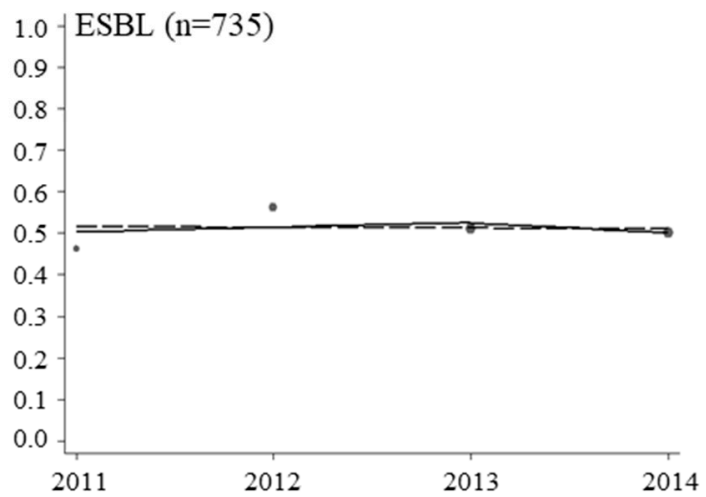

Year

Figure A2. Proportions of Klebsiella spp. isolates non-susceptible to cephalosporins and carbapenems, and extended-spectrum betalactamase (ESBL)-producing isolates from inpatients (A) and outpatients (B). The data were fitted with a piece-wise linear logistic regression model (solid line) and multivariable regression spline procedure (MVRS) (dashed line). Each bubble shows the proportion of isolates non-susceptible to the specific antibacterial category or the proportion of ESBL-producing isolates for the years 2011-2014. The size of the bubbles represents the number of isolates tested per year relative to the total number of isolates tested $(n)$ (see Table A2). $n=$ total number of isolates tested for the specific antibacterial category or ESBL-production in the whole period. 
Table A1. Proportions (\%) of non-susceptible or ESBL-producing E. coli and Klebsiella spp. isolates and total number ( $n$ ) of isolates tested according to antibacterial category, ESBL production and year.

\begin{tabular}{|c|c|c|c|c|c|c|c|c|c|c|c|c|c|c|c|c|c|c|c|c|c|c|}
\hline \multirow{2}{*}{ Organism } & \multicolumn{2}{|c|}{2004} & \multicolumn{2}{|c|}{2005} & \multicolumn{2}{|c|}{2006} & \multicolumn{2}{|c|}{2007} & \multicolumn{2}{|c|}{2008} & \multicolumn{2}{|c|}{2009} & \multicolumn{2}{|c|}{2010} & \multicolumn{2}{|c|}{2011} & \multicolumn{2}{|c|}{2012} & \multicolumn{2}{|c|}{2013} & \multicolumn{2}{|c|}{2014} \\
\hline & $\%$ & $n$ & $\%$ & $n$ & $\%$ & $n$ & $\%$ & $n$ & $\%$ & $n$ & $\%$ & $n$ & $\%$ & $n$ & $\%$ & $n$ & $\%$ & $n$ & $\%$ & $n$ & $\%$ & $n$ \\
\hline \multicolumn{23}{|l|}{ E. coli } \\
\hline Cephalosporins & 81.5 & 119 & 84.2 & 133 & 78.4 & 171 & 80.1 & 246 & 88.6 & 429 & 87.4 & 650 & 100 & 28 & 88.2 & 721 & 92.7 & 904 & 91.4 & 1115 & 86.1 & 1248 \\
\hline Carbapenems & 8.1 & 62 & 0 & 38 & 5.6 & 90 & 7.3 & 109 & 25.1 & 195 & 57.8 & 263 & 58.8 & 17 & 74.6 & 627 & 76.9 & 849 & 68.1 & 1086 & 45.1 & 1228 \\
\hline ESBL-positive & $\mathrm{N} / \mathrm{A}$ & $\mathrm{N} / \mathrm{A}$ & $\mathrm{N} / \mathrm{A}$ & $\mathrm{N} / \mathrm{A}$ & 42.4 & 151 & 57.7 & 222 & 57.9 & 411 & 85.9 & 325 & 85.7 & 21 & 41.1 & 626 & 38.3 & 874 & 40.8 & 1067 & 46.2 & 1218 \\
\hline \multicolumn{23}{|l|}{ Klebsiella spp. } \\
\hline Cephalosporins & 90.4 & 52 & 88.2 & 76 & 86 & 93 & 89.3 & 140 & 90.4 & 230 & 90.8 & 381 & 100 & 34 & 90.4 & 518 & 91.5 & 649 & 92.6 & 881 & 84.9 & 819 \\
\hline Carbapenems & 12.1 & 33 & 10.3 & 29 & 7.4 & 54 & 17.5 & 97 & 39.4 & 170 & 60 & 270 & 46.7 & 30 & 84.1 & 483 & 79.8 & 639 & 69.2 & 858 & 60.6 & 800 \\
\hline ESBL-positive & $\mathrm{N} / \mathrm{A}$ & $\mathrm{N} / \mathrm{A}$ & N/A & $\mathrm{N} / \mathrm{A}$ & 45.1 & 82 & 78.0 & 127 & 63 & 227 & 92.3 & 220 & 93.1 & 29 & 66.7 & 468 & 62.7 & 641 & 60 & 838 & 62.7 & 790 \\
\hline
\end{tabular}

ESBL: extended-spectrum betalactamase. $n=$ total number of isolates tested for the specific antibacterial category or ESBL-production in the specific year. 
Table A2. Proportions (\%) of non-susceptible or ESBL-producing E. coli and Klebsiella spp. isolates according to antibacterial category, ESBL production and year.

\begin{tabular}{|c|c|c|c|c|c|c|c|c|c|c|}
\hline \multirow{2}{*}{$\begin{array}{l}\text { Patient } \\
\text { Type }\end{array}$} & \multirow{2}{*}{ Organism } & \multirow{2}{*}{$\begin{array}{c}\text { Antibacterial } \\
\text { Category/ESBL }\end{array}$} & \multicolumn{2}{|c|}{2011} & \multicolumn{2}{|c|}{2012} & \multicolumn{2}{|c|}{2013} & \multicolumn{2}{|c|}{2014} \\
\hline & & & $\%$ & $n^{1}$ & $\%$ & $n^{1}$ & $\%$ & $n^{1}$ & $\%$ & $n^{1}$ \\
\hline \multirow{6}{*}{ Inpatient } & \multirow{3}{*}{ E. coli } & Cephalosporins & 90.4 & 428 & 94.7 & 527 & 91.6 & 618 & 90.0 & 712 \\
\hline & & Carbapenems & 77.7 & 386 & 78.9 & 512 & 74.3 & 610 & 49.1 & 707 \\
\hline & & ESBL-positive & 44.4 & 369 & 44.0 & 511 & 47.2 & 589 & 49.4 & 693 \\
\hline & \multirow{3}{*}{$\begin{array}{l}\text { Klebsiella } \\
\text { spp. }\end{array}$} & Cephalosporins & 94.2 & 345 & 95.6 & 410 & 94.3 & 527 & 90.3 & 524 \\
\hline & & Carbapenems & 91.0 & 333 & 87.0 & 414 & 75.0 & 519 & 68.5 & 523 \\
\hline & & ESBL-positive & 77.2 & 316 & 71.7 & 406 & 71.1 & 501 & 71.6 & 503 \\
\hline \multirow{6}{*}{ Outpatient } & \multirow{3}{*}{ E. coli } & Cephalosporins & 86.1 & 223 & 92.1 & 280 & 91.6 & 417 & 81.5 & 455 \\
\hline & & Carbapenems & 71.2 & 191 & 77.6 & 259 & 60.9 & 404 & 39.4 & 444 \\
\hline & & ESBL-positive & 36.8 & 193 & 31.5 & 267 & 34.5 & 400 & 42.4 & 448 \\
\hline & \multirow{3}{*}{$\begin{array}{l}\text { Klebsiella } \\
\text { spp. }\end{array}$} & Cephalosporins & 86.7 & 98 & 89.4 & 160 & 91.2 & 260 & 77.5 & 244 \\
\hline & & Carbapenems & 71.1 & 90 & 72.9 & 155 & 65.0 & 251 & 47.8 & 230 \\
\hline & & ESBL-positive & 46.2 & 91 & 56.1 & 157 & 51.0 & 249 & 50.0 & 238 \\
\hline
\end{tabular}

${ }^{1} n=$ total number of isolates tested. ESBL: extended-spectrum betalactamase.

Table A3. Odds ratios (OR) and 95\% confidence intervals (CI) of non-susceptible isolates from inpatients and outpatients for every year increment during the years 2011-2014 from a piece-wise logistic regression model.

\begin{tabular}{|c|c|c|c|c|c|c|c|}
\hline \multirow{3}{*}{$\begin{array}{l}\text { Patient } \\
\text { Type }\end{array}$} & \multirow{3}{*}{ Organism } & \multirow{3}{*}{$\begin{array}{l}\text { Antibacterial } \\
\text { Category/ESBL }\end{array}$} & \multicolumn{5}{|c|}{ Linear Trend } \\
\hline & & & \multicolumn{2}{|c|}{ Before the Knot } & \multirow{2}{*}{$\begin{array}{l}\text { Knot } \\
\text { Year }\end{array}$} & \multicolumn{2}{|c|}{ After the Knot } \\
\hline & & & OR & $95 \%$ CI & & OR & $95 \% \mathrm{CI}$ \\
\hline \multirow{6}{*}{ Inpatient } & \multirow{3}{*}{ E. coli } & Cephalosporins & 1.05 & $0.84-1.32$ & 2013 & 0.69 & $0.40-1.17$ \\
\hline & & Carbapenems & 0.90 & $0.77-1.04$ & 2013 & 0.36 & $0.26-0.50$ \\
\hline & & ESBL-positive & 0.99 & $0.77-1.28$ & 2012 & 1.12 & $0.81-1.56$ \\
\hline & \multirow{3}{*}{$\begin{array}{l}\text { Klebsiella } \\
\text { spp. }\end{array}$} & Cephalosporins & 0.99 & $0.73-1.34$ & 2013 & 0.53 & $0.27-1.04$ \\
\hline & & Carbapenems & 0.58 & $0.37-0.90$ & 2012 & 1.03 & $0.60-1.75$ \\
\hline & & ESBL-positive & 0.74 & $0.53-1.02$ & 2012 & 1.35 & $0.89-2.05$ \\
\hline \multirow{6}{*}{ Outpatient } & \multirow{3}{*}{ E. coli } & Cephalosporins & 1.32 & $1.01-1.72$ & 2013 & 0.28 & $0.15-0.52$ \\
\hline & & Carbapenems & 0.73 & $0.61-0.88$ & 2013 & 0.52 & $0.35-0.76$ \\
\hline & & ESBL-positive & 0.97 & $0.81-1.16$ & 2013 & 1.50 & $1.01-2.22$ \\
\hline & \multirow{3}{*}{$\begin{array}{l}\text { Klebsiella } \\
\text { spp. }\end{array}$} & Cephalosporins & 1.25 & $0.88-1.79$ & 2013 & 0.26 & $0.12-0.57$ \\
\hline & & Carbapenems & 0.83 & $0.64-1.07$ & 2013 & 0.57 & $0.34-0.97$ \\
\hline & & ESBL-positive & 1.05 & $0.83-1.32$ & 2013 & 0.86 & $0.53-1.42$ \\
\hline
\end{tabular}

ESBL: extended-spectrum betalactamase.

Table A4. Proportions (\%) of non-susceptible isolates according to specimen type and antibacterial category during the years 2004-2014, and proportions (\%) of ESBL-producing isolates according to specimen type during the years 2006-2014.

\begin{tabular}{ccccccc}
\hline Antibacterial & \multicolumn{3}{c}{ E. coli } & \multicolumn{3}{c}{ Klebsiella spp. } \\
\cline { 2 - 7 } Category/ESBL & Urinary & Other & $\boldsymbol{p}^{\mathbf{a}}$ & Urinary & Other & $\boldsymbol{p}^{\mathbf{a}}$ \\
\hline Cephalosporins & 85.2 & 92.0 & $<0.001$ & 86.8 & 91.2 & $<0.001$ \\
Carbapenems & 54.8 & 61.7 & $<0.001$ & 61.8 & 67.0 & 0.003 \\
ESBL-positive & 42.3 & 53.6 & $<0.001$ & 58.6 & 67.7 & $<0.001$ \\
\hline
\end{tabular}

${ }^{\text {a }} p<0.050$ (Chi square test, 2-tailed) indicates statistical significance of the differences in non-susceptibility rates. ESBL: extended-spectrum betalactamase. 
Table A5. Proportions (\%) of non-susceptible or ESBL-producing isolates according to gender and antibacterial category in the period 2004-2014, and according to gender and ESBL production in the period 2006-2014.

\begin{tabular}{ccccccc}
\hline Antibacterial & \multicolumn{3}{c}{ E. coli } & \multicolumn{3}{c}{ Klebsiella spp. } \\
\cline { 2 - 7 } Category/ESBL & Males & Females & $p^{\text {a }}$ & Males & Females & $p^{\text {a }}$ \\
\hline Cephalosporins & 91.8 & 84.7 & $<0.001$ & 91.3 & 85.7 & $<0.001$ \\
Carbapenems & 60.6 & 54.9 & $<0.001$ & 69.4 & 58.1 & $<0.001$ \\
ESBL-positive & 50.6 & 43.3 & $<0.001$ & 68.6 & 54.9 & $<0.001$ \\
\hline
\end{tabular}

a $p<0.050$ (Chi square test, 2-tailed) indicates statistical significance of the differences in non-susceptibility rates. ESBL: extended-spectrum betalactamase.

\section{References}

1. World Economic Forum. Global Risks 2013; World Economic Forum: Geneva, Switzerland, 2013.

2. The World Health Organization (WHO). Antimicrobial Resistance: Global Report on Surveillance; The World Health Organization (WHO): Geneva, Switzerland, 2014.

3. The World Health Organization (WHO). Global Action Plan on Antimicrobial Resistance; The World Health Organization (WHO): Geneva, Switzerland, 2015.

4. The World Health Organization (WHO). Meeting report: Global antimicrobial resistance surveillance system (GLASS). In Proceedings of the Technical Meeting on the Early Implementation Phase, Copenhagen, Denmark, 22-23 October 2015.

5. Alagesan, M.; Gopalakrishnan, R.; Panchatcharam, S.N.; Dorairajan, S.; Mandayam Ananth, T.; Venkatasubramanian, R. A decade of change in susceptibility patterns of Gram-negative blood culture isolates: A single center study. Germs 2015, 5, 65-77. [CrossRef] [PubMed]

6. Bajpai, T.; Pandey, M.; Varma, M.; Bhatambare, G.S. Prevalence of extended spectrum beta-lactamase producing uropathogens and their antibiotic resistance profile in patients visiting a tertiary care hospital in central India: Implications on empiric therapy. Indian J. Pathol. Microbiol. 2014, 57, 407-412. [CrossRef] [PubMed]

7. Gopalakrishnan, R.; Sureshkumar, D. Changing trends in antimicrobial susceptibility and hospital acquired infections over an 8-year period in a tertiary care hospital in relation to introduction of an infection control programme. J. Assoc. Physicians India 2010, 58, 25-31. [PubMed]

8. Gupta, A.; Sharma, S.; Arora, A.; Gupta, A. Changing trends of in vitro antimicrobial resistance patterns in blood isolates in a tertiary care hospital over a period of 4 years. Indian J. Med. Sci. 2010, 64, 485-492. [CrossRef] [PubMed]

9. Saravanan, R.; Raveendaran, V. Antimicrobial resistance pattern in a tertiary care hospital: An observational study. J. Basic Clin. Pharm. 2013, 4, 56-63. [CrossRef] [PubMed]

10. Sharma, N.; Gupta, A.K.; Walia, G.; Bakhshi, R. A retrospective study of the changing trends of antimicrobial resistance of Klebsiella pneumoniae isolated from urine samples over last 3 years (2012-2014). J. Nat. Sci. Biol. Med. 2016, 7, 39-42. [CrossRef] [PubMed]

11. Somashekara, S.C.; Deepalaxmi, S.; Jagannath, N.; Ramesh, B.; Laveesh, M.R.; Govindadas, D. Retrospective analysis of antibiotic resistance pattern to urinary pathogens in a Tertiary Care Hospital in South India. J. Basic Clin. Pharm. 2014, 5, 105-108. [CrossRef] [PubMed]

12. Gandra, S.; Mojica, N.; Klein, E.Y.; Ashok, A.; Nerurkar, V.; Kumari, M.; Ramesh, U.; Dey, S.; Vadwai, V.; Das, B.R.; et al. Trends in antibiotic resistance among major bacterial pathogens isolated from blood cultures tested at a large private laboratory network in India, 2008-2014. Int. J. Infect. Dis. 2016, 50, 75-82. [CrossRef] [PubMed]

13. Bac-Test Laboratory Web Page. Available online: http://www.indiamart.com/bac-test-laboratory/ (accessed on 27 March 2017).

14. Indian Census 2011. Available online: http://www.census2011.co.in/census/district/354-nashik.html (accessed on 27 March 2017).

15. The World Health Organization (WHO). WHONET Software. Available online: http:/ /www.whonet.org/ software.html (accessed on 27 March 2017). 
16. Blomberg, B.; Mwakagile, D.S.; Urassa, W.K.; Maselle, S.Y.; Mashurano, M.; Digranes, A.; Harthug, S.; Langeland, N. Surveillance of antimicrobial resistance at a tertiary hospital in Tanzania. BMC Public Health 2004, 4, 45. [CrossRef] [PubMed]

17. National Committee for Clinical Laboratory Standards. Methods for Dilution Antimicrobial Susceptibility Tests for Bacteria That Grow Aerobically, 10th ed.; Approved Standard M07-A6; National Committee for Clinical Laboratory Standards (NCCLS): Wayne, PA, USA, 2015.

18. Bauer, A.W.; Kirby, W.M.; Sherris, J.C.; Turck, M. Antibiotic susceptibility testing by a standardized single disk method. Am. J. Clin. Pathol. 1966, 45, 493-496. [PubMed]

19. Clinical and Laboratory Standards Institute. Performance Standards for Antimicrobial Susceptibility Testing, CLSI Documents M100-S14-M100-S24; Clinical and Laboratory Standards Institute (CLSI): Wayne, PA, USA, 2004-2014.

20. Magiorakos, A.P.; Srinivasan, A.; Carey, R.B.; Carmeli, Y.; Falagas, M.E.; Giske, C.G.; Harbarth, S.; Hindler, J.F.; Kahlmeter, G.; Olsson-Liljequist, B.; et al. Multidrug-resistant, extensively drug-resistant and pandrug-resistant bacteria: An international expert proposal for interim standard definitions for acquired resistance. Clin. Microbiol. Infect. 2012, 18, 268-281. [CrossRef] [PubMed]

21. Royston, P.; Sauerbrei, W. Multivariable modeling with cubic regression splines: A principled approach. Stata J. 2007, 7, 45-70.

22. Braykov, N.P.; Eber, M.R.; Klein, E.Y.; Morgan, D.J.; Laxminarayan, R. Trends in resistance to carbapenems and third-generation cephalosporins among clinical isolates of Klebsiella pneumoniae in the United States, 1999-2010. Infect. Control Hosp. Epidemiol. 2013, 34, 259-268. [CrossRef] [PubMed]

23. Jean, S.S.; Coombs, G.; Ling, T.; Balaji, V.; Rodrigues, C.; Mikamo, H.; Kim, M.J.; Rajasekaram, D.G.; Mendoza, M.; Tan, T.Y.; et al. Epidemiology and antimicrobial susceptibility profiles of pathogens causing urinary tract infections in the Asia-Pacific region: Results from the Study for Monitoring Antimicrobial Resistance Trends (SMART), 2010-2013. Int. J. Antimicrob. Agents 2016, 47, 328-334. [CrossRef] [PubMed]

24. Toner, L.; Papa, N.; Aliyu, S.H.; Dev, H.; Lawrentschuk, N.; Al-Hayek, S. Vancomycin resistant enterococci in urine cultures: Antibiotic susceptibility trends over a decade at a tertiary hospital in the United Kingdom. Investig. Clin. Urol. 2016, 57, 129-134. [CrossRef] [PubMed]

25. Pieboji, J.G.; Koulla-Shiro, S.; Ngassam, P.; Adiogo, D.; Njine, T.; Ndumbe, P. Antimicrobial resistance of Gram-negative bacilli isolates from inpatients and outpatients at Yaounde Central Hospital, Cameroon. Int. J. Infect. Dis. 2004, 8, 147-154. [CrossRef] [PubMed]

26. Saperston, K.N.; Shapiro, D.J.; Hersh, A.L.; Copp, H.L. A comparison of inpatient versus outpatient resistance patterns of pediatric urinary tract infection. J. Urol. 2014, 191, 1608-1613. [CrossRef] [PubMed]

27. National Centre for Disease Control. National Treatment Guidelines for Antimicrobial Use in Infectious Diseases, version 1.0; Directorate General of Health Services, Ministry of Health and Family Welfare, Government of India: New Dehli, India, 2016.

28. The World Health Organization (WHO). World Health Day 2011. Available online: http://www.who.int/ world-health-day/2011/en/ (accessed on 27 March 2017).

29. Indian Initiative of Management of Antibiotic Resistance Web Page. Available online: http:/ / save-antibiotics. blogspot.se/2011/04/activities-of-iimar-members-on-world.html (accessed on 27 March 2017).

30. Antibiotic Stewardship Network in India Web Page. Available online: http://www.absn.in/ (accessed on 27 March 2017).

31. Gjelstad, S.; Hoye, S.; Straand, J.; Brekke, M.; Dalen, I.; Lindbaek, M. Improving antibiotic prescribing in acute respiratory tract infections: Cluster randomised trial from Norwegian general practice (prescription peer academic detailing (Rx-PAD) study). Br. Med. J. 2013, 347. [CrossRef] [PubMed]

32. Stalsby Lundborg, C.; Tamhankar, A.J. Understanding and changing human behavior-Antibiotic mainstreaming as an approach to facilitate modification of provider and consumer behaviour. Upsala J. Med. Sci. 2014, 119, 125-133. [CrossRef] [PubMed]

33. Directorate General of Health Services. National Policy for Containment of Antimicrobial Resistance in India; Ministry of Health and Family Welfare, Government of India: New Dehli, India, 2011.

34. Wattal, C.; Raveendran, R.; Goel, N.; Oberoi, J.K.; Rao, B.K. Ecology of blood stream infection and antibiotic resistance in intensive care unit at a tertiary care hospital in North India. Braz. J. Infect. Dis. 2014, 18, $245-251$. [CrossRef] [PubMed] 
35. Lohiya, A.; Kant, S.; Kapil, A.; Gupta, S.K.; Misra, P.; Rai, S.K. Pattern of Antibiotic Resistance Among Community Derived Isolates of Enterobacteriaceae Using Urine Sample: A Study from Northern India. J. Clin. Diagn. Res. 2015, 9. [CrossRef] [PubMed]

36. European Centre for Disease Prevention and Control. Antimicrobial Resistance Surveillance in Europe 2014. Annual Report of the European Antimicrobial Resistance Surveillance Network (EARS-Net); European Centre for Disease Prevention and Control (ECDC): Stockholm, Switzerland, 2015.

37. Behera, B.; Mathur, P.; Das, A.; Kapil, A. Ertapenem susceptibility of extended spectrum beta-lactamase-producing Enterobacteriaceae at a tertiary care centre in India. Singap. Med. J. 2009, 50, 628-632.

38. Singh, A.K.; Jain, S.; Kumar, D.; Singh, R.P.; Bhatt, H. Antimicrobial susceptibility pattern of extended-spectrum beta-lactamase producing Klebsiella pneumoniae clinical isolates in an Indian tertiary hospital. J. Res. Pharm. Pract. 2015, 4, 153-159. [CrossRef] [PubMed]

2018 by the authors. Licensee MDPI, Basel, Switzerland. This article is an open access article distributed under the terms and conditions of the Creative Commons Attribution (CC BY) license (http:/ / creativecommons.org/licenses/by/4.0/). 